\title{
Hierarchical approach to water body status misclassification
}

\author{
M. Loga \\ Faculty of Environmental Engineering, \\ Warsaw University of Technology, Poland
}

\begin{abstract}
Within the context of assessing status of water bodies in EU countries, the Water Framework Directive (WFD) has introduced notions of confidence, precision and probability of misclassification (PoM). Although defined by WFD in a rather vague manner, the three measures of uncertainty have become compulsory elements of the reporting process. The EC requires all classifications of European water bodies to be accompanied by estimates of these uncertainty measures. The article describes the Hierarchical Approach introduced to assess PoM of riverine water bodies' status using uncertain water quality monitoring data. The approach stems from the observation that uncertainty of higher level classifications (e.g. assessment of water body status) depends on uncertainties of lower order assessments, (e.g. assessments of chemical status and ecological status of that water body). Specifically, the Hierarchical Approach describes how uncertainties intrinsic for water quality measurements propagate through the stages of water body status classification. To assess PoM of a water body, twodimensional probability distributions are used sequentially. At every stage, they are derived by combining one-dimensional probabilities of committing statistical errors of the II-nd type when classifying corresponding elements of the lower stage. For instance, to assess PoM of status of some water body two onedimensional PoMs of its chemical and biological status are used. The proposed method of assessing PoM is also shortly discussed within the context of risks involved in water management decisions based on misclassified water bodies.
\end{abstract}

Keywords: water body status, surface waters monitoring, classification, confidence, precision, probability of misclassification, Hierarchical Approach. 


\section{Introduction}

The assessment of a water body status according to the Water Framework Directive (WFD) is a set of well defined procedures in which water quality monitoring data gathered for all European water bodies are processed resulting in assigning to each water body (WB) a unique number - its class. It was also expected by the European Commission that these procedures would be incorporated into the national water legislation in all EU countries (CIS [1]). This has already happened and at present the requirement of classification of water bodies in EU countries is followed in a rather strict manner. Within the context of assessing classes of water bodies in the EU countries, the WFD has introduced also notions of confidence, precision and probability of misclassification (PoM) of the class assessment. Although defined by the WFD in a rather vague manner, the three measures of uncertainty have become compulsory elements of the reporting process. This triggered a pan-European research aiming at formulation of precise definitions of the three types of uncertainty (Ellis and Adraenssens [2], Ellis [3]). To this end, shortly after Poland's accession to the EU, the country water bodies have been delimited and a debate opened on finding the reference water bodies for 26 types of rivers. Eventually Poland, as other European countries, adopted for referencing a group of water bodies, in which water quality and biological life can be considered by experts as 'showing no, or only very minor, evidence of distortion'. Through special measuring campaigns the experts designed class limits for integrated biological indicators EQR (Ecological Quality Ratio) as well as its parameters E0 and E1 (E0 - value of metric for which $\mathrm{EQR}=0, \mathrm{E} 1$ - a reference condition value).

As to confidence, precision and probability of misclassification it has become clear from the very beginning that the three uncertainty measures should characterize the quality of classification procedure for the group of water bodies rather than for individual WBs. The group approach is now considered in Poland as the only feasible. A similar approach is also adopted by other latecomers into the European Community. The reason for choosing such approach is clearly a practical one - statistical samples of the WFD-compatible water quality data collected for water bodies in these countries are not large enough for making any sensible inference or reliable estimates of the three uncertainty measures for any individual WB. It is especially true for biological data which at present are very scarce. By creating pooled sets of water quality data for groups of WBs of the same type, the three uncertainties can be estimated effectively and serve for assessing quality of the classification procedures.

The EC requires all classifications of the European water bodies to be accompanied by the three uncertainty measures. According to the WFD confidence is a probability that actual, though unknown, value of some statistical parameter (e.g. mean value of some water quality indicator) lies within some confidence interval, i.e. within some range around a specified number calculated from a set of measured values of the indicator (e.g. around the arithmetic mean from the statistical sample). Precision is usually taken as a number representing half of that range. The two definitions are basically sufficient to estimate 
confidence and precision using well known concepts of statistics either frequentionistic or Bayesian.

Probability of misclassification, the subject of this article, is slightly more complex. A beneath approach, called the Hierarchical Approach, is theoretically proposed for estimating probability of misclassification. The approach stems from the observation that uncertainty of classification at the highest level (e.g. when classifying a WB status) depends on uncertainties in lower level classifications, (e.g. when classifying individual indicators, when classifying chemical or ecological status of a WB, etc). Specifically, the Hierarchical Approach describes theoretically how uncertainties inherent for measurements of water quality indicators propagate through the stages of water body classification.

\section{Sources of uncertainty in surface waters classification}

Although procedures for the water bodies classification specified by the WFD are deterministic, a random character of the water monitoring data causes that the ultimate outcome of these procedures - status of a water body, requires probabilistic interpretations. Uncertainty associated with a status of a WB results from two primary factors - randomness of aquatic processes and inherent uncertainties of water quality measurements. The assessment of a water body status according to the WFD is a set of well defined procedures used for comparing water quality monitoring data with WB status class limits specified by experts. The task of assessing of the accompanying uncertainties is definitely more complex since it involves description (or a model) of propagation of all types of uncertainties through the procedural steps of water bodies classification. Sources of these uncertainties are described below.

It is randomness of the environmental processes and measuring procedures that primarily and directly affects monitoring data and causes uncertainty of numbers that are subsequently used for assessing status of a water body. A random course of external aquatic processes within a water body at points in which measurements are made or water samples taken is definitely a primary source of uncertainty. Hydrological and hydromorphological processes are the examples of such external processes. Also, instruments or sensors used for in situ point measurements are primary sources of uncertainty (e.g. Ingersoll [4]). In this instance data resulting from measurements are directly affected by the internal instrumental noise generated by thermal, electrical and other processes within the sensors. These effects are normally encapsulated by the notion of the instrumental accuracy. Also inaccuracies in performing manual or automated procedures of water or biological specimens sampling as well as uncertainties as to the conditions in which the samples are transported, preserved and stored are to be considered primary sources of the uncertainty. Finally, when water samples or biological specimens are examined in the laboratory, the uncertainty of the numerical outcomes of the analyses depends on instrumental and procedural uncertainties of the applied measuring devices and analytical methods. It is assumed that for any water quality indicator " $i$ " of type "w" all uncertainties 
resulting from the primary sources can be encapsulated within one integrated measure - the indicator's standard deviation $\sigma_{i}^{w}$. It is also assumed that standard deviation $\sigma_{i}^{w}$ can be estimated sufficiently well with the empirical standard deviation $\hat{\sigma}_{i}^{w}$. Letter "w" stands for "chemical", "physicochemical", "biological" or "hydromorphological".

To the secondary sources of uncertainty belong all procedures/methods of data aggregation. The instance of such aggregate is the estimate of the mean value of a water quality indicator at given measuring point, e.g. the arithmetic mean of the indicator values measured during some period $\mathrm{T}_{\mathrm{o}}$ at that point. Another example are spatial aggregates, like the arithmetic mean of the indicator values measured at $m$ measurement points of given water body at some instant of time or during specified period of time. The uncertainties generated by the secondary sources depend on the way the monitoring data are aggregated. They are also linked with the primary sources of uncertainties. Statistical measures for the uncertainties from the secondary sources are described in a paragraph on "class generator".

There exists also a special type of measurement data uncertainty - the sampling error, which results from measurements of water quality indicators in discrete moments of time. Since in the natural environment the indicators evolve continuously in time, the discrete sampling causes a loss of information, hence an error. Another source of uncertainty is a part of the classification procedure that incorporates the experts' judgments. The example is uncertain character of class limits introduced by experts indirectly when defining the reference data for the water quality indicators. Although interesting itself and very likely important, discussion on data uncertainties resulting from the sampling error or the experts' judgment is omitted in this article.

Further on, a simplifying assumption is made according to which the uncertainties in monitoring data resulting from the primary sources (local environmental conditions and point measurements) are considered much smaller than uncertainties generated from the secondary sources. This intuitive statement comes from the observation that contemporary water quality monitoring systems are neither sufficiently dense in space nor measure water quality frequently enough to reflect on space and time variability of inland aquatic systems with sufficient accuracy. As mentioned before, uncertainties from the primary sources are characterized by statistical estimates of standard deviations of individual indicators. They can be inserted into the uncertainties resulting from the secondary sources in a natural way (see the next paragraph) making the uncertainties from the secondary sources a suitable departure point for assessing uncertainty of the WB status.

\section{Class of water body status}

This paragraph contains algorithms for classifying a WB status at some arbitrary measuring point with respect to the "w"-type group of indicators. It is assumed that all monitoring data necessary for the classification are available for the purpose. Definitions and procedures refer to the set of monitoring data consisting 
of values of "w-type" indicators that have been measured and collected within periods $\mathrm{T}_{1}, \mathrm{~T}_{2}, \ldots . \mathrm{T}_{\mathrm{No}}$ at given measuring point. Here, the EQR values for biological elements are considered to represent measured indicators for group "w" = "biological". The following notations have been assumed:

$\mathrm{N}_{\mathrm{w}}$ - number of measured variables within the group of "w"-type indicators.

$\underline{x}_{i}^{w}=\left(x_{i 1}^{w}, x_{i 2}^{w}, \ldots, x_{i T(w, i)}^{w}\right)-$ vector of $\mathrm{T}(\mathrm{w}, i)$ measured values of $i$-th indicator of ,w”-type, $\left(i=1, \ldots, \mathrm{N}_{\mathrm{w}}\right)$.

$\underline{\bar{x}}^{w}=\left(\bar{x}_{1}^{w}, \bar{x}_{2}^{w}, \ldots, \bar{x}_{N_{w}}^{w}\right)$-vector of the estimates of mean values of $\mathrm{N}_{\mathrm{w}}$ indicators of type "w".

$c l_{i}^{w},\left(i=1, \ldots, \mathrm{N}_{\mathrm{w}}\right)$ - numbers of the classes to which the estimates of mean values of indicators of type " $w$ " do belong.

$e^{w}=\min \left\{c l_{i}^{w}: i=1, \ldots, N_{w}\right\}-$ number of class to which a water body status belongs with respect to "w"-type indicators.

Algorithm for classifying a given WB status at some measuring point with respect to the "w"-type group of indicators can be summarized as follows:

1. Values of the estimates of mean values for indicators of type "w" $-\bar{x}_{i}^{w},(i=$ $\left.1, \ldots, N_{w}\right)$ are calculated directly from given monitoring data $\underline{x}_{i}^{w}=$ $\left(x_{i 1}^{w}, x_{i 2}^{W}, \ldots, x_{i T(w, i)}^{W}\right)$

$$
{\overline{x_{i}}}^{w}=\frac{1}{T(w, i)} \sum_{t=1}^{t=T(w, i)} x_{i t}{ }^{w},\left(i=1, \ldots, \mathrm{N}_{\mathrm{w}}\right)
$$

2. Number of a class, $c l_{i}^{w},\left(i=1, \ldots, \mathrm{N}_{\mathrm{w}}\right)$ to which the estimate of the average value of the $i$-th indicator of type "w" does belong can be found by inspection. Numbers that enable the classification for indicators are defined by the national Water Laws. In particular, the range of values the i-th indicator of type "w" may attain is subdivided into $K_{i}^{w}$ classes, $(i=1, \ldots$, $\left.\mathrm{N}_{\mathrm{w}}\right)$. For each class $k,\left(k=1,2, \ldots K_{i}^{w}\right)$ values of the lower class limit $L_{i}^{w}(k)$ and the upper class limit $U_{i}^{w}(k)$ are specified and published. For some indicators there is no upper or lower limits. In such instances $U_{i}^{w}=+\infty$ or $L_{i}^{w}=-\infty$ are conveniently assumed. Number of the class $\kappa \equiv c l_{i}^{w}$ for the $i$-th indicator of type " $\mathrm{w}$ " can be found by observing that a number $\kappa$, corresponding to given estimate of mean value $-\bar{x}_{i}^{w}$, is the one satisfying inequality

$$
L_{i}^{w}\left(\kappa \equiv c l_{i}^{w}\right) \leq \bar{x}_{i}^{w}<U_{k}^{w}\left(\kappa \equiv c l_{i}^{w}\right),\left(i=1, \ldots, \mathrm{N}_{\mathrm{w}}\right)
$$

Remark: for biological quality elements indicators their membership to one of the five classes is established from their EQR values.

3. Overall class $e^{w}$ to which a water body belongs with respect to indicators of "w"-type - is established by applying the One-Out-All-Out (OOAO) principle, i.e.

$$
e^{w}=\min \left\{c l_{i}^{w}: i=1, \ldots, N_{w}\right\}
$$




\section{Class generator $G\left(w, e^{w}\right)$ for water quality indicators}

Although assigning a class for a given WB with respect to indicators of "w"-type is not particularly complex, still a task of assessing the corresponding uncertainties requires processing water quality monitoring data in some ordered and consistent manner. Firstly, a concept of "class generator for water quality indicators that belong to group $w "-G\left(w, e^{w}\right)$, is introduced as a short hand notion substituting of otherwise lengthy descriptions of classification procedures. In short, the class generator is a name for all mathematical and logical operations performed on water quality monitoring data concerning indicators of group "w" that produces one number - an overall class $\mathrm{e}^{\mathrm{w}}$ to which water body status belongs with respect to given group of indicators. For any classification level two (or more) class generators of type $\mathrm{G}\left({ }^{*}, e^{*}\right)$ are specified and ultimately combined producing one class of a WB for that level. It is tacitly assumed that all classifications considered and analyzed in this article are referenced to the specified period $T_{0}$ in which water quality indicators have been measured at given water body as a part of national water monitoring program. Likewise, it is assumed that all considerations refer to some fixed water body or to fix measuring point within a water body.

\section{The three measures of uncertainty}

This paragraph contains algorithms for assessing the three uncertainties confidence, precision and probability of misclassification in a mode of classification in which water quality data are used directly. As before it is assumed that all monitoring data necessary for estimating the uncertainties are available for the purpose. Definitions and procedures refer to the set of monitoring data consisting of values of "w-type" indicators that have been measured and gathered within periods $\mathrm{T}_{1}, \mathrm{~T}_{2}, \ldots . \mathrm{T}_{\mathrm{No}}$ at given measuring point.

Algorithm for estimating first two measures of uncertainties - confidence and precision can be summarized in three simple steps:

1. The first measure of uncertainty - confidence, is estimated here after making a simplifying assumption about distribution of true value of the indicators' mean values and using classical formulae to estimate the length of the interval. The measure of uncertainty $\delta \bar{x}_{i}^{w}$ for $i$-th indicator of "w"-type , $(i=$ $1, \ldots, N_{w}$ ) can be calculated in three steps.

i) it is assumed that the indicator can be described as a random variable having Normal Probability Density function (p.d.f.).

ii) it is assumed that from measuring data gathered within a given observation period $\mathrm{T}_{\mathrm{o}}$, the estimate of standard deviation $\hat{\sigma}_{i}^{W}$ is close to standard deviation $\sigma_{i}^{w}$ in statistical population of values attained by the indicator. 
iii) it is estimated at the $90 \%$ level of confidence that true value of the mean value of the indicator lies within the range

$$
\left(\bar{x}_{i}^{w}-1.65 \frac{\sigma_{i}^{w}}{\sqrt{T(w, i)}}, \bar{x}_{i}^{w}+1.65 \frac{\sigma_{i}^{w}}{\sqrt{T(w, i)}}\right)
$$

This range is considered to be a measure of confidence.

2. Another measure of uncertainty related to the $i$-th indicator of "w"-type , $(i=$ $\left.1, \ldots, N_{w}\right)$ is its precision. It is defined as a half of the length of confidence interval (4), i.e.

$$
\delta \bar{x}_{i}^{w}=1.65 \frac{\sigma_{i}^{w}}{\sqrt{T(w, i)}},\left(\mathrm{i}=1, \ldots, \mathrm{N}_{\mathrm{w}}\right)
$$

3. Finally, as a measure of precision of classification $-\delta e^{w}$, of status of given WB with respect to group of indicators of "w"-type precision of "the worst" indicator class is assumed, i.e.

$$
\delta e^{w}=1.65 \frac{\sigma_{i_{o}}^{w}}{\sqrt{T\left(w, i_{o}\right)}}
$$

where $i_{\mathrm{o}}$ is a number of the indicator for which OOAO principle does hold, i.e. $c l_{i_{o}}^{w}=e^{w}$.

Algorithm for assessing probability of misclassification is more complex. The difficulty arises from the fact that the estimate of the mean for the $i$-th indicator of group "w" $-\bar{x}_{i}^{w}$ (used for the class assessment), is a random variable attaining its random realizations with the probability distribution specific for the given indicator. Although probability distribution of $\bar{x}_{i}^{w}$ has smaller standard deviation - formula (6), than standard deviation of the indicator, actual mean for the $i$-th indicator may belong to the class which is different than the class pointed by $\bar{x}_{i}^{w}$. This is the misclassification problem. Below two symmetric formulae, (7) and (8), are given for probabilities of misclassification of a water body in two cases:

a) when the assessment made with the use of the class generator $\mathrm{G}\left(\mathrm{w}, e^{w}\right)$ points class "+" which is higher than actual class membership of the water body status and

b) when the assessment made with the use of the class generator $\mathrm{G}\left(\mathrm{w}, e^{w}\right)$ points class "-" which is lower than actual class membership of the water body status.

In this article classical definition of probability of misclassification is adopted. It is assumed to be identical with probability of committing an error of the II-nd type, i.e. of accepting (not rejecting) statistical hypothesis $H_{o}$ when the hypothesis is false:

$$
\rho=P(\text { II-nd type error })=P\left(\text { not rejecting } H_{o} \text { when } H_{o} \text { is false }\right) \text {. }
$$


Hypothesis $H_{o}$ is related to class assessment of WB status and, in case a), can be formulated as follows: $\mathrm{H}_{0}=$ WB status class is assessed as "+". Alternative statistical hypothesis is $\mathrm{H}_{1}=$ actual WB status belongs to class "-". By frequentionistic interpretation $\rho$ is the probability that generator $\mathrm{G}\left(\mathrm{w}, e^{w}\right)$ when processing (random) measuring data of $i_{0}$-th indicator of group "w" within a given WB falsely assesses the class of the WB status as "+", while the actual WB status belongs to class "-". In one-dimensional case this probability can be estimated as

$$
\rho(+\mid-)=\frac{N\left(e^{w} \rightarrow "+" \mid e^{o}="-"\right)}{N\left(e^{o}="-"\right)}
$$

where

$N\left(e^{w} \rightarrow "+\mid e^{o}="-"\right)$ - number of cases (monitoring periods) for which generator $\mathrm{G}\left(\mathrm{w}, \mathrm{e}^{\mathrm{w}}\right)$ operating on values of $i_{0}$-th indicator of group " $\mathrm{w}$ " measured within periods $\mathrm{T}_{1}, \mathrm{~T}_{2}, \ldots . \mathrm{T}_{\mathrm{No}}$ assessed a class of WB as "+" while the WB belonged to class "_"

$N\left(e^{o}="-"\right)$-overall number of cases (monitoring periods), in which WB belonged to class "-"

$N_{o}-\quad$ total number of monitoring periods $\mathrm{T}_{1}, \mathrm{~T}_{2}, \ldots . . \mathrm{T}_{\mathrm{No}}$, in which the values of $i_{0}$-th indicator of group "w" were measured within given WB.

Similarly, the probability that generator $\mathrm{G}\left(\mathrm{w}, \mathrm{e}^{\mathrm{w}}\right)$ when processing (random) measuring data of $i_{0}$-th indicator of group "w" within a given WB falsely assesses the class of the water body status as "-", while the WB belongs to class "+" can be estimated as

$$
\rho(-\mid+)=\frac{N\left(e^{w} \rightarrow "-" \mid e^{o}="+"\right)}{N\left(e^{o}="+"\right)}
$$

where $N\left(e^{w} \rightarrow "-" \mid e^{o}="+"\right)$ - number of cases (monitoring periods) for which generator $\mathrm{G}\left(\mathrm{w}, \mathrm{e}^{\mathrm{w}}\right)$ operating on values of $i_{0}$-th indicator of group " $w$ " measured within periods $\mathrm{T}_{1}, \mathrm{~T}_{2}, \ldots . \mathrm{T}_{\mathrm{No}}$ assessed a class of WB status as "-", while the true WB status belonged to class "+".

$N\left(e^{o}="+"\right)$ - overall number of cases (monitoring periods), in which WB status belonged to class "+".

Expression $\mathrm{e}^{\mathrm{o}}=$ " $+"$ " (respectively, $\mathrm{e}^{\mathrm{o}}=$ " -") can be interpreted in two ways:

i) either, "correct" assessment of WB $-e^{o}$ is "+" (respectively, "-")

ii) or, the assessment made in the reference WB-ies for given type of WB indicates class "+" (respectively "“"). Class assessed for the reference WB-ies 
is operationally assumed to be the "correct" assessment $e^{o}$. In literature this assessment is called "expected class".

Case i) is purely theoretical since in real situations "true" class is not known.

Case ii) is the one which is of practical interest. It needs emphasising that probability of misclassification is in fact a measure of quality of the generator in making correct classification of WBs. Small and moderate values of this probability make the classifications assigned by the generator more reliable. When the probability of misclassification is large, the class generator is likely useless and needs exchanging for another generator.

\subsection{Example}

Formula (8) was used to calculate probability of misclassification for a simulated times series of EQR for phytobentos in a group of Malopolska province riverine water bodies of the $19^{\text {th }}$ type. Monte-Carlo simulator generated artificial (random) values of EQR with Normal p.d.f. of zero mean value and standard deviation equal to standard deviation of values of phytobentos EQR observed/measured in the rivers of this province. Length of generated long time series was $\mathrm{T}(\mathrm{w}, 0)=100000$. From this series $\mathrm{N}_{\mathrm{o}}=1000$ shorter random subseries have been chosen having their length $\mathrm{T}_{\mathrm{i}}=20$, $\left(\mathrm{i}=1, \ldots, \mathrm{N}_{\mathrm{o}}\right)$. For each subseries corresponding estimate ${\overline{x_{E Q R}}}^{w}$ of the mean value has been calculated from formula (1) which allowed to identify a class pointed by the class generator in a given period $T_{i},\left(i=1, \ldots, N_{o}\right)$. Since the true mean value fluctuates with Normal p.d.f. around the estimate ${\overline{x_{E Q R}}}^{w}$ with standard deviation $\frac{\sigma_{E Q R}^{w}}{\sqrt{T(w, E Q R)}}$ Monte-Carlo method was used to calculate a fraction of cases when "true" mean value has fallen to the lower class - formula (7) and similarly when it fallen into to higher class - formula (8).

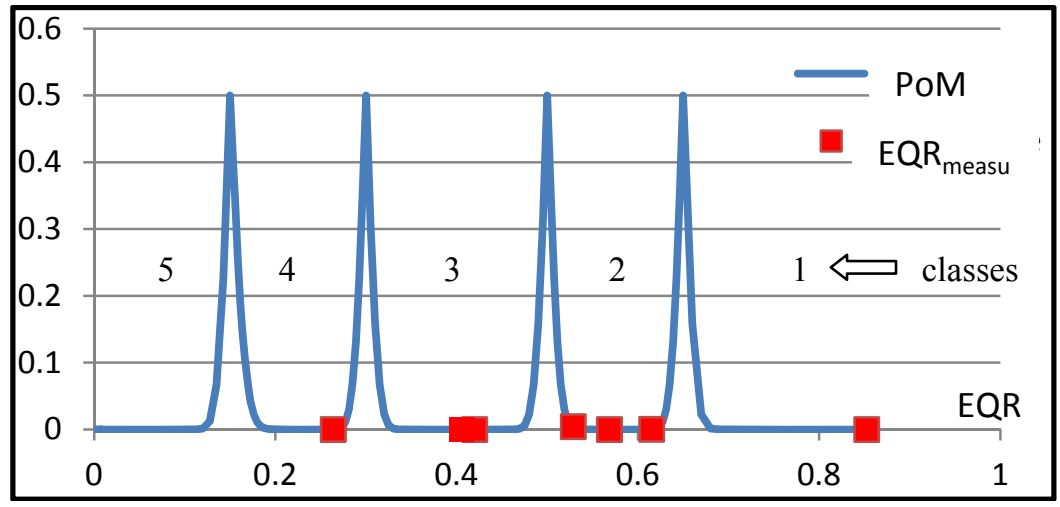

Figure 1: $\quad$ Probability of misclassification (PoM) for riverine water bodies in the Malopolska province in Poland for phytobentos EQR. 
Figure 1 shows how probability of misclassification is distributed for phytobentos EQR in the Malopolska province rivers. Squares represent seven riverine water bodies in the province having largest sets of phytobentos EQR data. The arithmetic mean of the pool sets of EQR values measured in these rivers show that probability of misclassification for these rivers is quite low.

\section{Hierarchy of the classification procedure}

Classification of water bodies in subsequent periods $T_{1}, T_{2}, \ldots$ is an important function of the water monitoring systems in all EU countries. According to the intention of the WFD, classification of water bodies status is the mechanism through which water management decisions can be guided to achieve and sustain good status of inland waters in Europe. For instance, when status of the water body deteriorates and becomes worse than "good" it is expected that some remediation measures are enforced by water management authorities and lead to "recovery" of the good status.

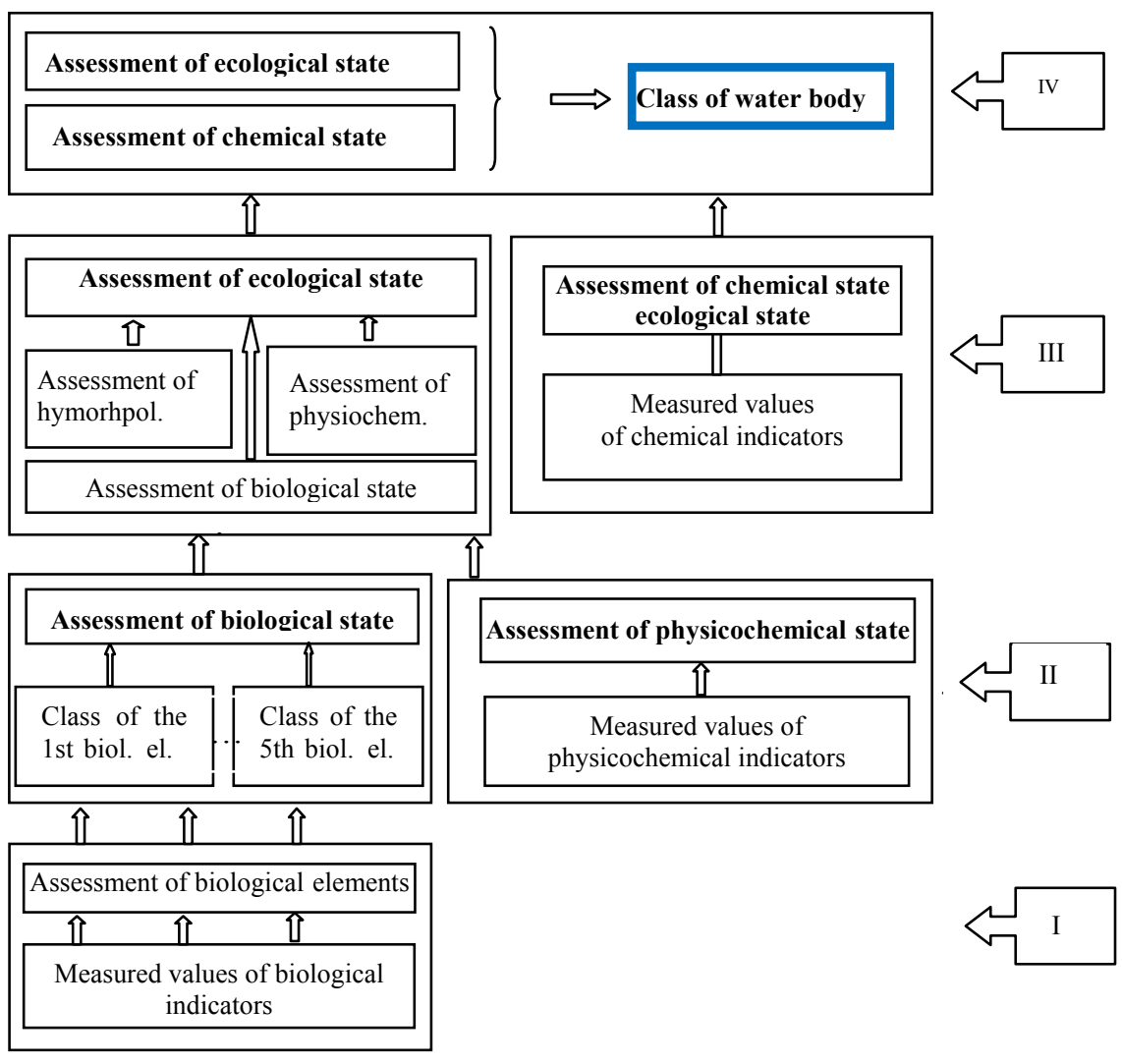

Figure 2: $\quad$ Hierarchical structure of WB status classification. 
The classification task is accomplished by processing water quality monitoring data within the hierarchical structure predefined by the WFD. There are four levels in the classification hierarchy. At the lower level-level I, the measured values of chemical, physicochemical, hydromorphological and biological indicators are classified. On the very top level-level IV, ultimate classification of a water body status is accomplished. The hierarchy is presented in Figure 2. It can be observed that there are two modes of classification-basic mode and WFD-mode. In the basic mode measured values of water quality indicators are directly used to classify a water body with respect to indicators of the "w"-type. Steps (1) and (2) of paragraph 3 are applied to every group "w" of indicators.

Class $e^{w}$ to which a water body belongs with respect to indicators of "w"type - is established by applying the One-Out-All-Out (OOAO) principle formula (3). This mode is applied on levels I, II and III when assessing biological, physicochemical and chemical states of water bodies. The classes $e^{w}$ established using the basic mode of classification are then processed through the second mode of classification - a WFD-mode. This mode consists of chain of logical rules defined by the WFD. It enables to classify ecological status and then, by combining it with chemical status, to assess a class of the water body (levels III and IV). It can be observed that all the classification rules of the WFDmode can be reduced to making choice between "upper class" (assigned as "+") and "lower class" (assigned as "-"). The choice is made by considering two lower level assessments made by two class generators $G\left(e_{1}\right)$ and $G\left(e_{2}\right)$. Generator $\mathrm{G}\left(\mathrm{e}_{1}\right)$ can point one of the two classes "+ ${ }^{\mathrm{el}}$ " or " _el". Similarly, generator $\mathrm{G}\left(\mathrm{e}_{2}\right)$ can point one of the two classes " ${ }^{\mathrm{e} 2}$ " or " $\mathrm{e}$ " ". According to the WFD-mode of classification the "upper class" is achieved only when both generators, $\mathrm{G}\left(\mathrm{e}_{1}\right)$ and $\mathrm{G}\left(\mathrm{e}_{2}\right)$, point their respective "upper classes", i.e. " $+{ }^{\mathrm{e} 1 "}$ " and " $+{ }^{\mathrm{e} 2}$ ". Otherwise the WFD- mode chooses the "lower class". This illustrates Figure 3. Task of estimating the three uncertainties required by the WFD can be simplified by

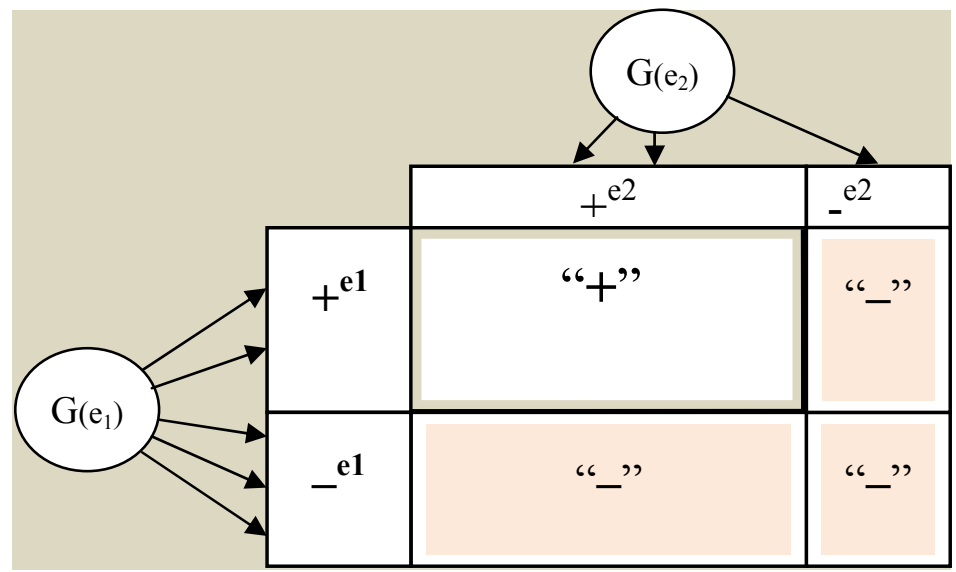

Figure 3: $\quad$ WFD-mode of classification. 
processing (propagating) elementary uncertainties of water quality indicators through hierarchy of procedural steps of water body classification. In the basic mode of classification confidence, precision and probability of misclassification can be estimated using formulae (5)-(8). Assessing the three uncertainties within the WFD-mode is more complex for it involves two-dimensional distributions of probability. As an example of the WFD-mode classification probability of misclassification is theoretically assessed in the next paragraph.

\section{Probability of misclassification within hierarchical classification structure}

When classifying ecological status or assessing class of a water body the WFDmode is applied (at levels III and IV of the hierarchical structure). For instance when assessing a class of a water body from ecological status and chemical status of that water body, general scheme presented in Figure 3 can be used for $\left(\mathrm{e}_{1}=\mathrm{e}^{\mathrm{ec}}\right)$ and $\left(\mathrm{e}_{2}=\mathrm{e}^{\mathrm{ch}}\right)$. Consequently, for assessing probability of misclassification, two-dimensional versions of formulae (7) and (8) must be followed:

$$
\begin{gathered}
\rho^{*}(+\mid-)=\frac{N\left(e^{e c} \rightarrow "+{ }^{e c} " \wedge e^{c h} \rightarrow "+{ }^{c h} " \mid e^{o}="-\right)}{N\left(e^{o}="-"\right)} \\
\rho^{*}(-\mid+)=\frac{N\left(\neg\left(e^{e c} \rightarrow "+^{e c "} \wedge e^{c h} \rightarrow "+{ }^{c h "}\right) \mid e^{o}="+"\right)}{N\left(e^{o}="+"\right)}
\end{gathered}
$$

where

$$
N\left(e^{e c} \rightarrow "+{ }^{e c "} \wedge e^{c h} \rightarrow "+{ }^{c h "} \mid e^{o}="-"\right) \text { - number of cases (monitoring }
$$

periods) for which generators $G^{\text {ec }}$ and $G^{\text {ch }}$ point at the upper ecological status " $+{ }^{\mathrm{ec} \text { " }}$ and at the upper chemical status " + ch" respectively, while true class of water body status $\mathrm{e}^{\mathrm{o}}$ is ".-".

$N\left(\neg\left(e^{e c} \rightarrow "{ }^{e c "} \wedge e^{c h} \rightarrow "+{ }^{c h "}\right) \mid e^{o}="+"\right)$ - number of cases (monitoring periods) for which at least one of the generators $\mathrm{G}^{\mathrm{ec}}$ or $\mathrm{G}^{\mathrm{ch}}$ points at its lower status "." (so, at "_ec" or "_ch"), while true class of water body status $\mathrm{e}^{\mathrm{o}}$ is " + ".

$N\left(e^{o} \rightarrow "+"\right)$ - overall number of cases (monitoring periods), in which both true ecological status and true chemical status belonged to their upper classes, i.e. when true WB status belonged to class "+".

$N\left(e^{o} \rightarrow "-"\right)=N_{o}-N\left(e^{o} \rightarrow "+"\right)-\quad$ overall number of cases

(monitoring periods) for which true WB status belonged to class “"”.

It needs to be remarked that generator $\mathrm{G}^{\text {ch }}$ points at chemical status of WB according to the basic mode, i.e. by operating on values of chemical indicators (priority substances) measured within periods $\mathrm{T}_{1}, \mathrm{~T}_{2}, \ldots . \mathrm{T}_{\mathrm{No}}$. This is different from generator $\mathrm{G}^{\mathrm{ec}}$, which points at ecological status of $\mathrm{WB}$ according to the 
WFD-mode. Clearly, generator $\mathrm{G}^{\mathrm{ec}}$ operates on generators that assess biological status $\left(G^{b}\right)$, physicochemical status $\left(G^{\text {pch }}\right)$, and hydromorphological status $\left(G^{\text {hm }}\right)$ each using basic mode of classification.

\section{Conclusions}

When introducing definitions of confidence, precision and probability of misclassification, the Water Framework Directive did not set numerical values for critical magnitudes of the three measures of uncertainty. This was the right decision for it could lead to not realistic requirements for the water quality monitoring systems in the EU countries. At the same time the key principle was introduced stating that proper level of confidence and precision should allow for adequate assessment of status of water bodies in the member countries. Possibly, the word "adequate" would mean that critical magnitudes for the three uncertainties were to be introduced by the EU countries themselves according to overall status of their water bodies. This freedom is contrasting with the envisaged strictness of checking by the EC if the national critical magnitudes for the uncertainties are suitable for imposing remediation measures in case when status of WB worsens. Definitely, such attitude, is "not adequate" to the present, rather low, level of scientific insight into the relationships between the detrimental changes in status of water bodies and costs of introducing suitable remediation measures. There is even less knowledge on assessing of how much it would cost to make an error of the II-nd type. Bearing this in mind this research has been restricted to much simpler issue of finding out how to assess the probability of misclassification of water body status within multilevel hierarchy of heuristic procedures imposed by the WFD. The basic mode of classification was applied to estimate probability of misclassification of $\mathrm{EQR}$ for practical case of water bodies in the Malopolska province in Poland. For the WFD-mode only theoretical considerations has been outlined. The envisaged next step will be to show applicability of the Hierarchical Approach to estimation of probability of misclassification of water bodies status by combining basic and WFD modes of classification for the same practical case of riverine water bodies in the Malopolska province in Poland.

\section{References}

[1] CIS, W. G. (2005). Common implementation strategy for the Water Framework Directive (2000/60/EC), Guidance Document No 13, Overall Approach to the Classification of Ecological Status and Ecological Potential.

[2] Ellis, J. and Adraenssens, V., Uncertainty estimation for monitoring results by the WFD biological classification tools. Environment Agency, 2006.

[3] Ellis J.E. Combining Multiple Quality Elements and Defining Spatial Rules for WFD Classification, Environment Agency, 2007.

[4] Ingersoll, W.S., Environmental Analytical Measurement Uncertainty Estimation. Nested Hierarchical Approach, Defense Technical Information Center, 2001. 